\title{
Análise da Incorporação de Requisitos não Funcionais na Modelagem de Processos de Negócio
}

\author{
Guilherme B. M. Salles, Marcelo Fantinato \\ Escola de Artes, Ciências e Humanidades - Universidade de São Paulo \\ São Paulo - SP - Brasil \\ \{guilherme.salles, m.fantinato\}@usp.br
}

\begin{abstract}
Considering the business process management, strategic alignment is important due to the need to manage and optimize IT processes starting from the organization's most important processes. In the requirements engineering phase of a IS development, not only functional requirements should be took into account, but also the operating and quality constraints that related processes should consider. This paper aims at, through a systematic analysis, presenting the existing research works linking business processes modeling to non-functional requirements. As a result, the published studies are discussed and some gaps into this research line are pointed.
\end{abstract}

Resumo. Considerando a gestão de processos de negócio, o propósito do alinhamento estratégico deve-se à necessidade de gerenciar e otimizar a TI a partir dos processos mais importantes à organização. Na fase de engenharia de requisitos de um SI, é importante atentar-se não só aos requisitos funcionais, mas também às restrições de operação e a qualidade que os processos devem considerar. $O$ presente artigo busca, por meio de uma análise sistemática, apresentar os artigos existentes para associar a modelagem de processos de negócio a requisitos não funcionais. Como resultado, tem-se a discussão dos estudos publicados para abordar esse tema e a observação de lacunas nessa linha de pesquisa.

\section{Introdução}

O cenário corporativo mundial acompanhou, nas últimas décadas, uma transformação importante no setor de Tecnologia da Informação (TI), em que esta se tornou objeto essencial às organizações e vem agregando valor ao negócio. Tal transição é justificada fundamentalmente pela competição existente no mercado, uma vez que a TI se tornou um meio de acesso e descoberta de informações precisas à tomada de decisões e, dependendo do negócio principal da organização, é indispensável que os serviços prestados aos clientes disponham de tecnologia [Laudon e Laudon 2009].

Nesse novo ambiente, organizações de diversos setores consideram imprescindível realizar investimentos expressivos em TI, a fim de obter produtos, serviços e processos apoiados nessa ferramenta. Assim, a necessidade das organizações serem competitivas frente às mudanças contínuas faz com que elas também se modifiquem e se planejem com o auxílio dos recursos da TI. Mais do que realizar uma escolha para aprimorar seu trabalho e buscar resultados eficientes, as organizações necessitam dessa contínua adaptação para sobreviver em um cenário tão concorrido. 
A questão estratégica de se integrar os recursos de TI ao negócio vem consumindo grande esforço humano e financeiro. Com essa necessidade emergente, as organizações devem ter os Planejamentos Organizacionais e de TI coerentes e com sinergia, viabilizando o alinhamento das estratégias de TI com as estratégias de negócio. Essa atividade é um desafio contínuo e evita que recursos investidos em TI sejam desperdiçados nas organizações por meio de baixa produtividade e má qualidade nos processos e serviços [Shimizu, Carvalho e Laurindo 2006].

Considerando a Gestão de Processos de Negócio (BPM - do inglês Business Process Management), o propósito do alinhamento estratégico deve-se à necessidade de gerenciar e otimizar a TI a partir dos processos mais importantes à organização. Nesse sentido, é importante atentar-se ao cumprimento dos requisitos não funcionais (NFR do inglês Non-Functional Requirements), que conferem qualidade e eficiência aos processos de negócio, indo além das atividades em si que devem ser realizadas.

Em meio a essas necessidades, a engenharia de requisitos (tanto funcionais quanto não funcionais) é um fator crítico de sucesso em projetos de desenvolvimento de Sistemas de Informação (SI). Uma elicitação de requisitos mal realizada pode levar ao fracasso completo do projeto, o que está geralmente relacionado à falta de compreensão do negócio pelos analistas de sistemas, à falta de entendimento dos objetivos do sistema e também à deficiência na comunicação entre os envolvidos ativamente no projeto.

Gestores de diferentes departamentos organizacionais têm procurado conhecer mais o valor estratégico da TI, considerando suas peculiaridades e melhores práticas de gestão. Essa situação reforça que esse conhecimento é essencial, pelo investimento que representa e pela dependência cada vez mais forte que as organizações têm em relação a TI. Em contrapartida, no cenário atual, boa parte das organizações ainda não possui uma evolução efetiva no aprimoramento de seus processos [Rezende 2008]. De acordo com Rezende (2008), a aplicação de metodologias à gestão estratégica de TI garante um caminho consistente ao uso da TI como diferencial competitivo. Além disso, torna-se possível a gestão integrada dos processos e serviços, ampliando a afinidade da área de tecnologia com as áreas de negócio. Segundo Caseau (2005), a necessidade de se ter maior agilidade e redução de custos nos negócios resulta numa demanda crescente por bons processos e serviços. Os clientes esperam maior agilidade e qualidade, ao passo em que os prestadores de serviços enfrentam grandes desafios para garanti-las.

Deve-se identificar, às funções de um determinado processo, todos os requisitos de qualidade em potencial. A noção desses requisitos de qualidade se relaciona com os NFR da Engenharia de Software. Para monitorá-los e cumpri-los de forma automatizada nos serviços, costuma-se usar os conceitos de Acordo em Nível de Serviço (SLA - do inglês: Service Level Agreement) e QoS (Qualidade de Serviço).

Segundo Bratanis, Dranidis e Simons (2010), para o aspecto do negócio, outro tipo de acordo é necessário para se captar as restrições de qualidade dos serviços em um nível mais alto. A partir de um conjunto de SLA, compõe-se um Acordo em Nível de Negócio, que recebe o acrônimo de BLA (do inglês: Business Level Agreement). Este tipo de acordo envolve os parceiros (cliente e fornecedor) participantes do processo.

Este artigo apresenta uma Revisão Sistemática (RS) sobre métodos e técnicas usados para associar modelagem de processos de negócio (doravante chamados apenas 
de processos, a fim de simplificação neste artigo), sobretudo BPMN (Business Process Model and Notation), a NFR. Além disso, são analisados trabalhos que relacionam essas linguagens a restrições de qualidade e SLA ou BLA.

O artigo está estruturado conforme a seguir: seção de metodologia; seções de resultados da RS, evidenciando dados quantitativos, discutindo textualmente os artigos extraídos e analisando-os de forma crítica; e, seções de conclusões e trabalhos futuros.

\section{Metodologia}

Como metodologia principal para esta pesquisa, uma RS foi realizada a fim de compreender os estudos desenvolvidos acerca do tema apresentado. RS são pesquisas rigorosas e indicadas para capturar estudos primários e secundários sobre determinado assunto, avaliando e discutindo as abordagens existentes [Kitchenham 2004].

De acordo com Biolchini et al. (2005), as fases fundamentais de uma RS são: planejamento, execução, e análise dos resultados. No planejamento, delimita-se o tema de estudo e elabora-se o protocolo de pesquisa (artigo discutido com mais detalhes a seguir). Na fase de execução, pesquisa-se, de fato, os artigos buscados e lista-se o resultado dessas buscas de forma estruturada. $\mathrm{Na}$ discussão dos resultados, finalmente, deve-se expor e analisar os artigos identificados por meio das pesquisas realizadas.

Diferentemente de uma revisão bibliográfica comum, em que não se costuma elaborar um protocolo de pesquisa, o qual permitiria a reprodução dos passos do estudo, a RS, portanto, possibilita que outro pesquisador avalie e adapte os padrões escolhidos às particularidades de seu trabalho [Biolchini et al. 2005].

Como parte do protocolo de pesquisa do presente trabalho, foram criadas strings de pesquisa nas línguas inglesa e portuguesa, buscando cercar o tema em estudo com o máximo de termos possível. Essas palavras-chave foram usadas nas bibliotecas digitais online ACM e IEEE e também nas bases eletrônicas indexadas DBLP, Elsevier Scopus e Google Scholar. Tendo em vista que essa última retorna muitos artigos não científicos e ainda não trouxe artigos novos quando se realizou pesquisas preliminares nas bases, optou-se posteriormente por descartá-la. Ajustes precisaram ser realizados nas strings de pesquisa, considerando os detalhes técnicos de configuração das máquinas de busca de cada ferramenta. Houve, em alguns casos, intersecção de resultados entre as ferramentas de busca e também entre as strings lançadas em uma mesma ferramenta. As strings genéricas são apresentadas na Tabela 1 .

Tabela 1. Strings de pesquisa do protocolo de busca da RS

\begin{tabular}{|l|}
\hline $\begin{array}{l}\text { (BPM* OR "activit* diagram*") AND ("non functional requirement*" OR "non-functional } \\
\text { requirement*") }\end{array}$ \\
\hline (BPM* OR "activit* diagram*") AND (QoS OR quality) \\
\hline (BPM* OR "activit* diagram*") AND (BLA OR "business level agreement*") \\
\hline (BPM* OR "activit* diagram*") AND (SLA OR "service level agreement*") \\
\hline (BPM* OR "diagrama* de atividade*") AND ("requisito* não funciona*") \\
\hline (BPM* OR "diagrama* de atividade*") AND (QoS OR qualidade) \\
\hline $\begin{array}{l}\text { (BPM* OR "diagrama* de atividade*") AND (BLA OR "business level agreement*" OR (acordo nível } \\
\text { negócio)) }\end{array}$ \\
\hline $\begin{array}{l}\text { (BPM* OR "diagrama* de atividade*") AND (SLA OR "service level agreement*" OR (acordo nível } \\
\text { serviço)) }\end{array}$ \\
\hline
\end{tabular}


O objetivo principal, com o protocolo de pesquisa e as conseqüentes strings de busca, era encontrar artigos que relacionassem a modelagem de processos de negócio, via BPMN ou Diagrama de Atividades da UML, com aspectos não funcionais de uma forma geral, representados mais especificamente por NFR ou BLA ou SLA ou QoS. Por este motivo, a Seção 4 deste documento detalha os artigos que buscaram tal correlação.

$\mathrm{O}$ protocolo de pesquisa considera artigos que foram publicados apenas nos últimos oito anos (como critério de inclusão), visto que, por se tratar de um tema que avança o estado da arte na modelagem de processos, deve-se analisar publicações mais recentes. Como critérios de exclusão, artigos que apresentassem resultados sem esclarecer os métodos usados ou artigos que relacionassem apenas aspectos no nível de serviço, ou seja, sem envolver processos, foram eliminados dos resultados.

Como critério para qualidade dos resultados obtidos, artigos deveriam ter sido publicados em conferências ou periódicos, e dissertações de mestrado e teses de doutorado deveriam ter sido aprovadas por banca examinadora.

Ao final dessas etapas, de 44 artigos inicialmente encontrados, 21 artigos foram selecionados, os quais foram analisados na íntegra. Em seguida, identificou-se o foco principal de cada artigo para classificá-los de forma exclusiva, com o intuito de mapear seus objetivos, permitindo a apresentação e discussão dos artigos de forma conjunta. Por restrições de espaço, não foi possível detalhar neste artigo todo o resultado da RS. Assim, a classificação proposta auxiliou na delimitação dos artigos aqui abordados.

\section{Visão Geral dos Artigos Selecionados}

Nesta seção, são apresentados dados gerais relacionados aos artigos analisados na RS, enquanto que o conteúdo propriamente dito desses artigos são discutidos na Seção 4.

Dentre os 21 artigos selecionados, há uma média de 2,66 autores por artigo, com moda de três autores, ocorrendo em nove dos artigos. Em relação ao idioma, um se encontra na língua portuguesa e os 20 restantes, na língua inglesa. Em relação ao período, a Figura 1 mostra a distribuição dos artigos selecionados em função do ano de publicação (linha azul). Nota-se também uma tendência de aumento nos anos mais recentes (linha laranja), com uma projeção linear para o próximo período (ano de 2012). Como uma informação importante para os pesquisadores da comunidade brasileira, a Figura 2 apresenta a distribuição do número de artigos de acordo com o índice Capes Qualis vigente da área de Ciência de Computação [Capes 2010]. Percebe-se uma concentração de artigos publicados em periódicos e conferências com índices B2 e B3.
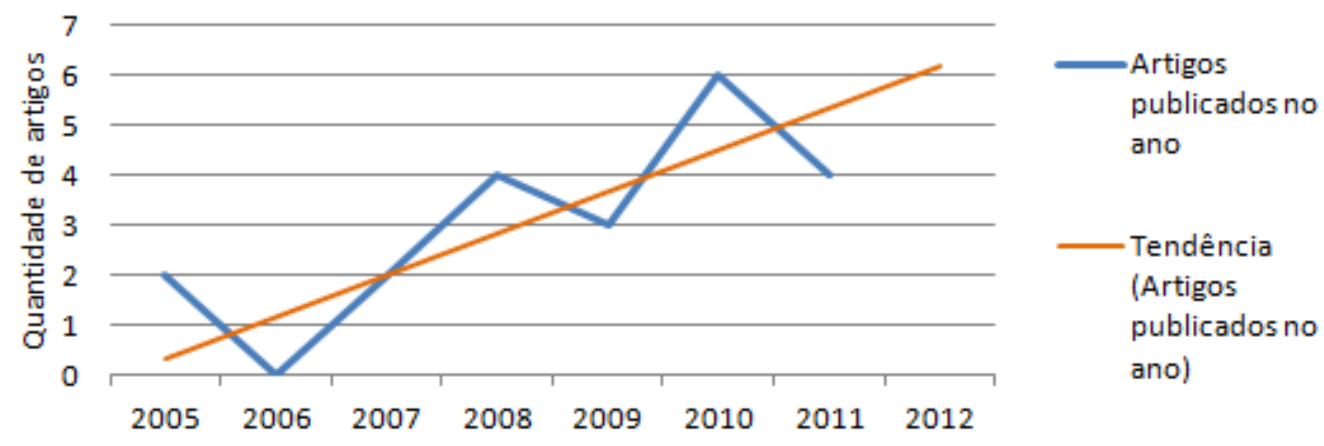

Figura 1. Distribuição dos artigos por ano de publicação 
Os 21 artigos selecionados foram classificados de forma exclusiva segundo seus principais focos de pesquisa, para descrevê-los e analisá-los de forma conjunta. A Tabela 2 apresenta as quatro classificações, que foram definidas e mapeadas durante a execução da RS, em função da análise dos próprios artigos selecionados e estudados.

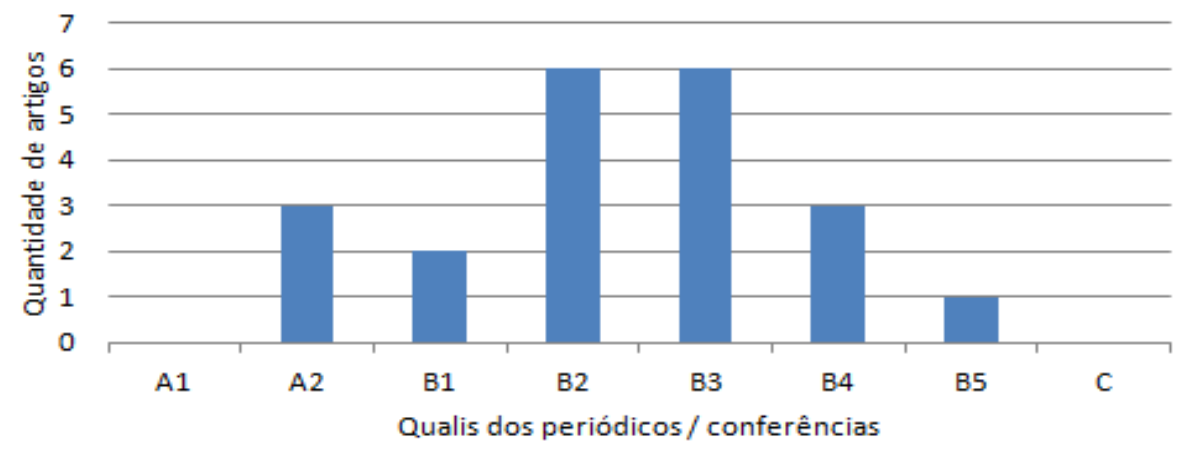

Figura 2. Distribuição dos artigos por índice Qualis dos periódicos e das conferências.

Tabela 2. Classificação dos artigos analisados na RS

\begin{tabular}{|c|c|c|}
\hline Classificação & Descrição & Artigos \\
\hline $\begin{array}{l}\text { 1. Extensão de } \\
\text { BPMN para NFR* }\end{array}$ & $\begin{array}{l}\text { Artigos que abordam } \\
\text { modelagem de processos } \\
\text { (BPMN ou Diagrama de } \\
\text { Atividades) associada à } \\
\text { representação de NFR }\end{array}$ & $\begin{array}{l}\text { 1. [Aburub, Odeh e Beeson 2007] } \\
\text { 2. [Khaluf, Gerth e Engels 2011] } \\
\text { 3. [Pavlovski e Zou 2008] } \\
\text { 4. [Saeedi, Zhao e Sampaio 2010] } \\
\text { 5. [Serrano, Caballero e García 2009] } \\
\text { 6. [Xavier et al. 2010] }\end{array}$ \\
\hline 2. SLA/QoS & $\begin{array}{l}\text { Artigos que abordam processos } \\
\text { e SLA (ou QoS) de forma } \\
\text { conjunta }\end{array}$ & $\begin{array}{l}\text { 1. [Beimborn e Joachim 2010] } \\
\text { 2. [Caseau 2005] } \\
\text { 3. [Forster, Engels e Schattkowsky 2005] } \\
\text { 4. [Haq, Huqqani e Schikuta 2009] } \\
\text { 5. [Theilmann et al. 2010] } \\
\text { 6. [Vujovic, Krivokapic e Sokovic 2011] }\end{array}$ \\
\hline 3. BLA & $\begin{array}{l}\text { Artigos que abordam processos } \\
\text { e BLA de forma conjunta }\end{array}$ & 1. [Bratanis, Dranidis e Simons 2010] \\
\hline $\begin{array}{l}\text { 4. Qualidade e } \\
\text { complexidade }\end{array}$ & $\begin{array}{l}\text { Artigos que tratam de métricas } \\
\text { de qualidade e complexidade } \\
\text { da modelagem de processos, } \\
\text { incluindo formas de avaliação } \\
\text { de modelos }\end{array}$ & $\begin{array}{l}\text { 1. [Bolloju e Sun 2008] } \\
\text { 2. [Cardoso 2007] } \\
\text { 3. [Heravizadeh, Mendling e Rosemann 2008] } \\
\text { 4. [Khlif e Makni 2009] } \\
\text { 5. [Sanchez et al. 2010] }\end{array}$ \\
\hline 5. Outros & $\begin{array}{l}\text { Artigos não classificados nas } \\
\text { demais categorias }\end{array}$ & $\begin{array}{l}\text { 1. [Jungen 2011] } \\
\text { 2. [Mazanek e Hanus 2011] } \\
\text { 3. [Vara, Sanchez e Pastor 2008] }\end{array}$ \\
\hline
\end{tabular}

\section{Descrição de Artigos Selecionados}

Nesta seção, são apresentados os artigos da classificação 1 da Tabela 2, ou seja, aqueles que propuseram extensões à modelagem de processos para contemplar aspectos de NFR. Esta RS buscou também artigos que abordassem modelagem de processos e SLA/BLA de forma conjunta, porém artigos encontrados envolvendo SLA/BLA não envolvem a modelagem de processos mas sim apenas processos de uma forma geral, conforme os itens 2 e 3 da Tabela 2, que por não serem foco principal do objetivo inicial desta RS não serão detalhados aqui, assim como os itens 4 e 5 da Tabela 2. 
Khaluf, Gerth e Engels (2011) introduzem uma nova forma de se modelar restrições de qualidade em processos. Segundo sua proposta, restrições de qualidade podem ser explicadas como relações temporais e lógicas que surgem em diferentes momentos nos processos. A qualidade do processo pode então ser verificada e medida conforme as restrições estabelecidas para ele. Para isso, uma nova linguagem para modelagem de restrições de qualidade é definida, embasada pelos padrões da Computation Tree Logic, que visa distribuir o tempo em ramos de árvores. Na abordagem proposta, processos são modelados em Diagramas de Atividades da UML 2.0 e restrições de qualidade são representadas por meio da PPSL (Process Pattern Language Specification), ambos de forma estendida. A EPPSL (Extended PPSL), uma nova linguagem de modelagem visual, correlaciona as restrições de qualidade da notação original em PPSL com os Diagramas de Atividades da UML. Estes, por sua vez, recebem dois novos símbolos para cobrir a semântica das restrições de qualidade, que não são definidas originalmente nos Diagramas de Atividades: o "guarda" - que indica, em cada atividade do processo, se há ou não restrições de qualidade; e, a "restrição de qualidade" - que indica quais as restrições presentes naquela determinada atividade. De acordo com os autores da proposta, a notação concebida traz flexibilidade aos usuários e analistas de sistemas para expressar NFR de processos.

Saeedi, Zhao e Sampaio (2010) sugerem que os requisitos de qualidade recebam atenção central nos negócios e na modelagem de processos, propondo a incorporação dos seguintes requisitos de qualidade à BPMN - tempo, custo e confiabilidade - por serem os preferidos de clientes e comumente abordados em SLA. Um meta-modelo foi elaborado para definir a associação entre tais requisitos e atividade do processo modelado. Esses requisitos são apresentados nos modelos de forma analítica e reduzida. A proposta estende a notação BPMN nos seguintes elementos: (i) ExtensionDefinition que define os atributos e os grupos da BPMN sofrendo modificações; (ii) ExtensionAttributeDefinition - que define os novos atributos que sendo incorporados à notação; e, (iii) ExtensionAttributeValue - que define os possíveis valores de cada atributo criado. Na Figura 3 está apresentado o resultado da extensão, contemplando graficamente os requisitos de tempo $(\mathrm{T}-$ time $)$, custo $(\mathrm{C}-$ cost $)$ e confiabilidade $(\mathrm{R}-$ reliability) no elemento atividade do processo. Esses requisitos podem ser ocultados na forma recolhida (collapsed) ou visualizados na forma expandida (expanded). Na Figura 4, é ilustrado um exemplo simples de processo em que os requisitos possuem valores. Os autores dessa proposta defendem que essa representação auxilia na especificação dos SLAs, porém não há automação dos requisitos criados à geração desses acordos.

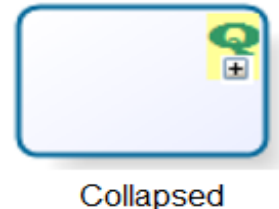

Collapsed

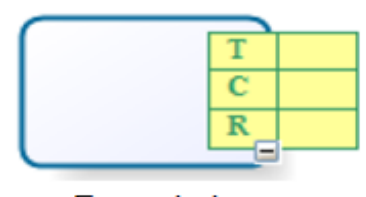

Expanded

Figura 3. Notação de atividade de BPMN com extensão para requisitos de qualidade [Saeedi, Zhao e Sampaio 2010].

De acordo com Serrano, Caballero e García (2009), muitas organizações têm percebido a importância cada vez maior de lidar com dados que tenham um nível adequado de qualidade para seus processos, evitando erros que possam trazer impactos negativos de desempenho. Vários artigos apresentam que, para alcançar esse objetivo, 
deve-se explicitar ao máximo os requisitos de Qualidade de Dados e de Informações e envolver a reengenharia dos processos da organização, mantendo atenção nos pontos mais críticos. Dentro desse contexto, BPMN fornece uma notação adequada para representar os processos, mas não para lidar com requisitos específicos de qualidade de dados. Por outro lado, IP-MAP (Information Products Maps) aborda esse quesito, embora não seja amplamente aceito para a modelagem de processos quanto BPMN, principalmente pela pouca oferta de ferramentas de apoio. Mapas IP-MAP permitem a especificação de processos por meio de um mapa conceitual, em que as atividades correspondentes à gestão da qualidade de dados são devidamente tratadas. Tendo observado a crescente demanda por ferramentas e técnicas para gerenciar requisitos de qualidade de dados, foi proposto então o preenchimento dessa lacuna, por acreditar ser necessário fornecer aos analistas de negócio fundamentos e ferramentas adequadas para tratar tais requisitos. Baseando-se em IP-MAP, o objetivo principal deste artigo foi analisar a capacidade da notação BPMN em representar as questões de Qualidade de Dados e Informações aos processos. Discutiu-se como estender BPMN para apoiar a qualidade de dados oferecida pelo IP-MAP, a fim de obter uma notação suficientemente rica. As estruturas de IP-MAP foram analisadas e mapeadas à notação BPMN. Como resultado, a abordagem proposta consiste na adição de um símbolo em forma de estrela (DQDim) que representa as dimensões da qualidade de dados controlada pelo processo.

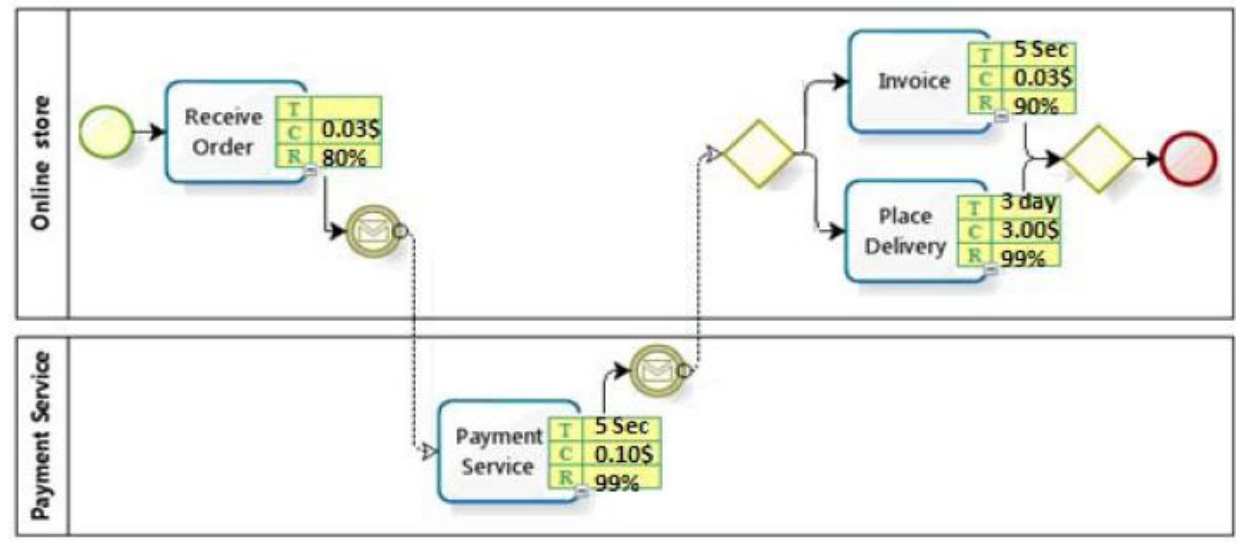

Figura 4. Exemplo do uso das atividades de BPMN com extensão para requisitos de qualidade [Saeedi, Zhao e Sampaio 2010].

Xavier et al. (2010) propõem a abordagem BPMNFR, que visa integrar a notação BPMN e NFR, descritos no NFR Framework, que consiste em um guia de requisitos não funcionais, tais como segurança, desempenho e custo, para direcionar o mapeamento dessas restrições em processos. No BPMNFR, novos símbolos são propostos para que a notação BPMN seja capaz de contemplar as devidas restrições NFR. Partindo de uma revisão acerca de trabalhos correlatos, a principal contribuição desse artigo é uma abordagem que orienta a descoberta dos NFR nos diagramas de processos, desde a criação do modelo em BPMN até o detalhamento dos NFR que devem ser inseridos nas atividades do diagrama. Além disso, tem-se a extensão do NFR Framework em relação à usabilidade, com o objetivo futuro de completar essa extensão para todo o catálogo usando os padrões da ISO/IEC 9126 (norma ISO para a qualidade de software). Embora oito requisitos tenham sido identificados - mobilidade, acessibilidade, usabilidade, auto-contenção, robustez, facilidade no transporte, baixo custo de manutenção, e baixo custo com infraestrutura - tratou-se apenas de usabilidade 
neste artigo, que visa introduzir a abordagem BPMNFR. A Figura 5 mostra um exemplo genérico de processo modelado em BPMN no qual determinadas atividades são especificadas com restrições de usabilidade, recebendo, para isso, o símbolo " $U$ ".

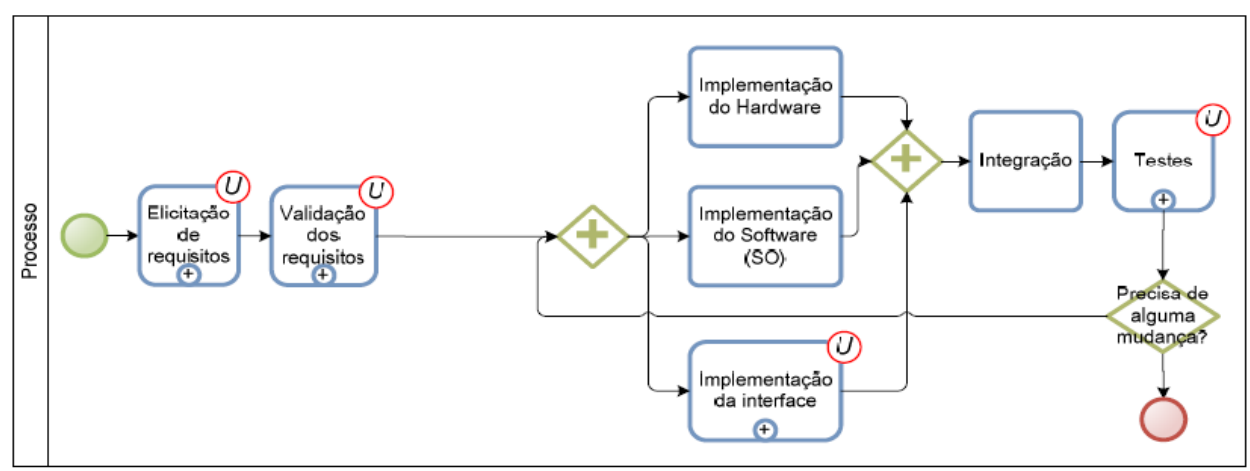

Figura 5. Extensão da notação BPMN proposta por Xavier et al. (2010)

Aburub, Odeh e Beeson (2007) trabalham com ideias do QFD (Quality Function Deployment), método capaz de mapear funções de qualidade de um produto ou serviço, e sugerem acoplá-lo à notação RAD (Role Activity Diagramming), usada para modelagem funcional de processos. Apesar de não abordar BPMN ou Diagrama de Atividades da UML, este artigo foi considerado na RS por tratar de modelagem de NFR em processos. Assim, foi desenvolvida uma abordagem em que NFR se associam ao modelo RAD do processo. Tem-se um casamento entre NFR e a representação funcional de RAD, uma vez que se identifica quais atividades, interações e funções do modelo funcional podem incorporar NFR. A Figura 6 ilustra o framework geral da abordagem, em que RAD e a análise de NFR são apresentados independentemente. A produção de NFR, nesse caso, é fundamentalmente um processo top-down, partindo-se da consideração de atributos gerais de qualidade do negócio. Esses atributos são vistos como "objetivos" ao negócio, a partir dos quais se derivam sub-objetivos. A modelagem de processos por meio de RAD, no entanto, se dá na direção bottom-up, identificando atividades da organização e representando-as como um conjunto de interações.

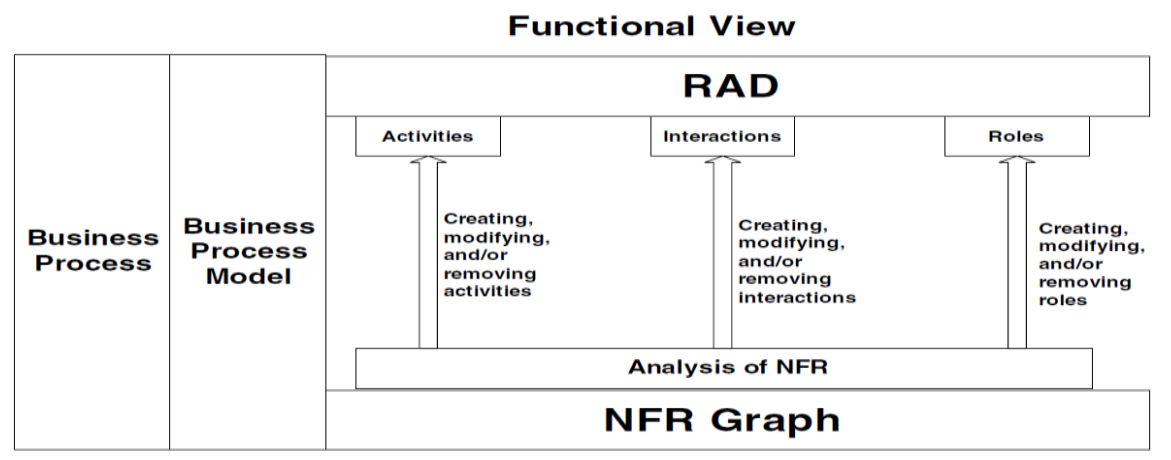

Non-Functional View

Figura 6. Associação das visões funcional (RAD) e não funcional (NFR Graph) [Aburub, Odeh e Beeson 2007].

Pavlovski e Zou (2008) propõem dois novos artefatos à BPMN para modelar NFR de processos. Conforme apresentado na Figura 7, o primeiro - operating condition - indica apenas a existência de determinada restrição associada a uma atividade, e o segundo - control case - define critérios de controle para mitigar o risco associado ao 
primeiro. Esse controle se dá por meio de três propriedades: descrição do risco associado à operating condition, descrição dos possíveis impactos para o negócio em que a tarefa está envolvida, e descrição dos controles que devem ser aplicados para mitigar ou anular os riscos. Essa extensão da BPMN permite que tanto interesses inerentes ao negócio quanto a NFR sejam modeladas adequadamente. Obtêm-se, portanto, um complemento semântico do fluxo do processo. Porém, assim como visto nos demais estudos correlatos, a identificação e modelagem dos NFR se limita ao alto nível de representação, não havendo decomposição da ideia às camadas de execução do processo, tampouco à associação dessas restrições a BLA e SLA a serem estabelecidos.

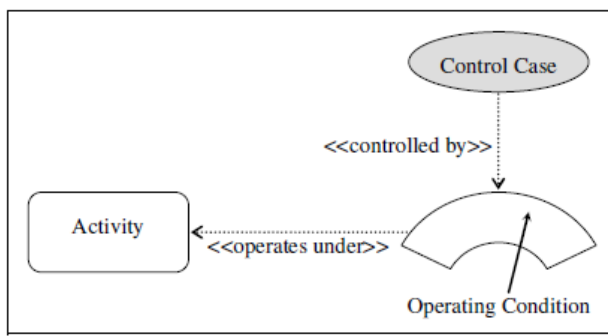

Figura 7. Novos artefatos para modelar NFR em BPMN [Pavlovski e Zou 2008].

\section{Discussão dos Resultados}

Características não funcionais são consideravelmente mais difíceis de se capturar na modelagem dos processos, já que o foco dessa atividade está na representação do comportamento funcional. As organizações costumam concentrar seus esforços para atender ao máximo os requisitos funcionais, mas a questão da qualidade ganha cada vez mais espaço quando se busca bons produtos e serviços, o que traz vantagem competitiva. O controle da qualidade interna dos processos, aos olhos da gestão de operações, determina a percepção externa da qualidade dos bens e serviços concebidos. Com isso, métodos que representem esses atributos de forma explícita se tornam necessários para, inclusive, apoiar a melhoria contínua dos processos, já que se explicita as atividades com restrições e necessidades específicas de qualidade.

De acordo com Heravizadeh, Mendling e Rosemann (2008), a dimensão da qualidade dos processos é normalmente negligenciada durante a modelagem. $\mathrm{O}$ foco tradicional de BPMN baseia-se em refletir as atividades sob o ponto de vista "as-is" que apenas captura a dimensão processual e fornece informações limitadas sobre fatores de qualidade envolvidos. Como consequência, a lacuna entre o "as-is" e o "to-be" é mal apoiada pelas ferramentas de modelagem de processo analisadas nesta RS.

Além disso, a dificuldade em compreender e automatizar de forma adequada os modelos de processos é parcialmente justificada pela ausência de tratamento dos NFR. Embora haja alguns artigos correlacionando restrições de qualidade e modelagem de processos (e recomendando essa associação), não foi encontrado qualquer modelo para transcrever esses requisitos em BLA ou SLA à fase de execução. Analisando os artigos avaliados que buscaram estender linguagens de modelagem de processos, todos limitaram-se à representação gráfica de NFR, sem que os símbolos adicionados à notação influenciem em qualquer automação do processo representado. Essa possível transcrição automática dos requisitos incorporados à modelagem permitiriam não só a execução do processo (em linguagem WS-BPEL, por exemplo), mas também a 
formalização de BLA ou SLA a padrões tais como WS-Agreement ou WS-Policy. Além disso, os artigos publicados até o momento incorporaram NFR a cada atividade do modelo de processos, e não a um conjunto de atividades ou ao processo como um todo. Se esta associação fosse realizada, a geração de BLA seria bastante facilitada. O resultado observado relaciona estes NFR com SLA, uma vez que os BLA costumam ser agregações hierárquicas de SLA em um nível mais alto.

Os benefícios apontados sobre a modelagem de NFR no contexto aqui abordado são, principalmente, a vantagem de visualizar adequadamente as características não funcionais dos processos. Além disso, torna-se possível detectar as restrições de um determinado SI ainda na fase de elicitação de requisitos, o que, naturalmente, reduz despesas em relação à identificação tardia das mesmas. Há também a facilidade imediata de se identificar potencias SLA e BLA que devem ser estabelecidos posteriormente quando os NFR já se encontram mapeados no modelo. Quanto a esses acordos, os benefícios de sua criação aos processos estão no ganho da qualidade da execução dos processos e o alinhamento formal entre as expectativas dos clientes (negócios) e as obrigações dos fornecedores (TI). A importância de se formalizar tais acordos deve-se também à dificuldade de verificar a qualidade de processos quando estes são complexos ou quando têm suas restrições formuladas em textos informais.

\section{Conclusão}

A modelagem de processos é uma etapa de grande importância dentro do ciclo de vida de BPM. Apesar de pesquisas apontarem a importância e os benefícios de se associar formalmente atividades e processos a NFR, tais como tempo, custo e disponibilidade, as linguagens de modelagem analisadas são pobres nessa questão. Isto, conforme apresentado, pode ser crítico para o sucesso de projetos de desenvolvimento de SI.

Com esta RS, identificou-se iniciativas para a extensão de linguagens de modelagem de processos, tais como BPMN, Diagramas de Atividades da UML e RAD, visando representar requisitos de qualidade ou restrições de execução. Porém, as propostas existentes não se aprofundam à automação desses requisitos à fase de execução do processo, salvo quando mencionado como trabalhos futuros. Além disso, todas essas pesquisas incorporam NFR a cada atividade do modelo, o que dificulta a relação dessas restrições com a geração de BLA (em geral, um conjunto de SLA). Ou seja, a transformação de BPMN (linguagem de modelagem em nível abstrato padrão) para BPEL (linguagem de execução em nível de codificação padrão) é bem conhecida e aceita, mas a automação de NFR em BLA e SLA ainda é pouco explorada.

Como resultado prático desta RS, pretende-se estudar a lacuna acima apresentada e propor uma extensão à BPMN suficientemente rica para contemplar a representação de BLA e a automação desses NFR ao nível de execução dos processos.

\section{Agradecimentos}

Este trabalho foi financiado parcialmente pela Fapesp (Fundação de Amparo à Pesquisa do Estado de São Paulo). Os professores doutores Edmir P. V. Prado, Fátima L. S. N. Marques e João P. de Albuquerque contribuíram para a realização deste trabalho. 


\section{Referências}

Aburub, F., Odeh, M. and Beeson, I. (2007) "Modelling non-functional requirements of business processes", Information and Software Technology, Ed. Elsevier, 49 edition, p. 1162-1171.

Beimborn, D. and Joachim, N. (2010) "The joint impact of service-oriented architectures and business process management on business process quality: an empirical evaluation and comparison", Information Systems and E-Business Management, p. 333-362.

Biolchini, J., Mian, G., Natali, A. C. and Travassos, G. (2005) "Systematic Review in Software Engineering", Technical Report RT-ES 679/05, System Engineering and Computer Science Dept., COOPE/UFRJ, 30 p.

Bolloju, N. and Sun, S. (2008) "Exploiting the Complementary Relationship between Use Case Models and Activity Diagrams for Developing Quality Requirements Specifications", In: 27th Int. Conf. on Conceptual Modeling, p. 144-153.

Bratanis, K., Dranidis, D. and Simons, A. (2010) "Towards run-time monitoring of business-level agreements for web services", In 5th Annual South-East European Doctoral Student Conf. (SEERC), Thessaloniki, p. 370-379.

Capes. (2010) “Ciência da Computação: Documento de Área - 2009”, Coordenação de Aperfeiçoamento de Pessoal de Nível Superior - Diretoria de Avaliação, http://www.capes.gov.br/images/stories/download/avaliacao/COMPUTACAO_05ma r10.pdf, Dezembro.

Cardoso, J. (2007) "Business Process Quality Metrics: Log-Based Complexity of Workflow Patterns", Lecture Notes in Computer Science - Springer, p. 427-434.

Caseau, Yves. (2005) "Self-Adaptive Middleware: Supporting Business Process Priorities and Service Level Agreements", Advanced Engineering Informatics. Ed. Elsevier, $19^{\text {th }}$ edition, p. 199-211.

Forster, A., Engels, G. and Schattkowsky, T. (2005) "Activity Diagram Patterns for Modeling Quality Constraints in Business Processes”, ACM/IEEE Int. Conf. on Model-Driven Engineering Languages and Systems (MoDELS), p. 2-16.

Haq, I., Huqqani, A. and Schikuta, E. (2009) "Aggregating hierarchical service level agreements in business value networks", In: BPM Workshops - Int. Conf. on Business Process Management Workshops, p. 176-192.

Heravizadeh, M., Mendling, J. and Rosemann, M. (2008) "Dimensions of Business Processes Quality (QoBP)", In: 6th Int. Conf. on Business Process Management Workshops (BPM Workshops), Milan, Italy, p. 80-91.

Jungen, M. (2011) "Stakeholder-Driven Business Process Management - An Evaluation of the Suitability, Adequacy and Effectiveness of Quality and Process Management", Int. Conf. on Human-Computer Interaction (HCI), p. 244-252.

Khaluf, L., Gerth, C. and Engels, G. (2011) "Pattern-Based Modeling and Formalizing of Business Process Quality Constraints", Int. Conf. on Advanced Information Systems Engineering (CAiSE), p. 521-535. 
Khlif, W. and Makni, L. (2009) "Quality metrics for business process modeling”, In: Int. Conf. on Applied Computer Science, Genova, p. 195-200.

Kitchenham, B. (2004) "Procedures for Performing Systematic Reviews", Joint Technical Report, Keele University and Empirical Software Engineering National ICT Australia Ltd., 28 p.

Laudon, K. C. and Laudon, J. P. (2009), Management Information Systems, PrenticeHall, $11^{\text {th }}$ edition.

Mazanek, S. and Hanus, M. (2011) "Constructing a bidirectional transformation between BPMN and BPEL with a functional logic programming language", Journal of Visual Languages and Computing, Ed. Elsevier, $22^{\text {th }}$ edition, p. 66-89.

Pavlosvki, C. J. and Zou, J. (2008) "Non-functional Requirements in Business Process Modeling", In: 15th Asia-Pacific Conf. on Conceptual Modelling (APCCM), Computer Society Inc., Darlinghurst, Australia, p. 103-112.

Rezende, D. A. (2008), Tecnologia da Informação e Planejamento Estratégico, Brasport, $1^{\text {st }}$ edition.

Saeedi, K., Zhao, L. and Sampaio, P. R. F. (2010) "Extending BPMN for Supporting Customer-Facing Service Quality Requirements", IEEE Int. Conf. on Web Services (ICWS), p. 616-623.

Sanchez, L., García, F., Mendling, J. and Ruiz, F. (2010) "Prediction of Business Process Model Quality based on Structural Metrics", In: 29th Int. Conf. on Conceptual Modeling (ER), Vancouver, Canada, p. 458-463.

Serrano, N. S., Caballero, I. and García, F. (2009) "Extending BPMN to Support the Modeling of Data Quality Issues", Int. Conf. on Inform. Quality (ICIQ), p. 46-60.

Shimizu, T., Carvalho, M. and Laurindo, F. (2006), Strategic Alignment Process and Decision Support Systems: Theory and Case Studies, IRM Press, $1^{\text {st }}$ edition.

Theilmann, W., Winkler, U., Happe, J. and Magrans, I. (2010) "Managing on-demand business applications with hierarchical service level agreements", Lecture Notes in Computer Science (LNCS) - Springer, p. 97-106.

Vara, J., Sanchez, J. and Pastor, O. (2008) "Business Process Modelling and Purpose Analysis for Requirements Analysis of Information Systems", Springer-Verlag, Berlin, Heidelberg, p. 213-227.

Vujovic, A., Krivokapic, Z. and Sokovic, M. (2011) "Improvement of Business Processes Performances through Establishment of the Analogy: Quality Management System - Human Organism”, Journal of Mechanical Engineering, p. 151-161.

Xavier, L., Alencar, F., Castro, J. and Pimentel, J. (2010) "Integração de Requisitos Não-Funcionais a Processos de Negócio: Integrando BPMN e NFR", In: 13th Workshop em Engenharia de Requisitos (WER), Cuenca, Ecuador, p. 29-40. 